\title{
CD36 deficiency impairs intestinal lipid secretion and clearance of chylomicrons from the blood
}

\author{
Victor A. Drover, ${ }^{1}$ Mohammad Ajmal, ${ }^{1}$ Fatiha Nassir, ${ }^{2}$ Nicholas O. Davidson, ${ }^{3}$ \\ Andromeda M. Nauli, ${ }^{4}$ Daisy Sahoo, ${ }^{5}$ Patrick Tso, ${ }^{4}$ and Nada A. Abumrad ${ }^{1,2}$
}

1Department of Physiology and Biophysics, State University of New York at Stony Brook, Stony Brook, New York, USA. ²Department of Medicine, Division of Nutrition and ${ }^{3}$ Division of Gastroenterology, Washington University, St. Louis, Missouri, USA. ${ }^{4}$ Department of Pathology and Laboratory Medicine, University of Cincinnati, Cincinnati, Ohio, USA. 5Department of Pharmacology, State University of New York at Stony Brook, Stony Brook, New York, USA.

\begin{abstract}
CD36 mediates the transfer of fatty acids (FAs) across the plasma membranes of muscle and adipose cells, thus playing an important role in regulating peripheral FA metabolism in vivo. In the proximal intestine, CD36 is localized in abundant quantities on the apical surface of epithelial cells, a pattern similar to that of other proteins implicated in the uptake of dietary FAs. To define the role of CD36 in the intestine, we examined FA utilization and lipoprotein secretion by WT and CD36-null mice in response to acute and chronic fat feeding. CD36-null mice given a fat bolus by gavage or fed a high-fat diet accumulated neutral lipid in the proximal intestine, which indicated abnormal lipid processing. Using a model in which mice were equipped with lymph fistulae, we obtained evidence of defective lipoprotein secretion by directly measuring lipid output. The secretion defect appeared to reflect an impaired ability of CD36-null enterocytes to efficiently synthesize triacylglycerols from dietary FAs in the endoplasmic reticulum. In the plasma of intact mice, the reduced intestinal lipid secretion was masked by slow clearance of intestine-derived lipoproteins. The impaired clearance occurred despite normal lipoprotein lipase activity and likely reflected feedback inhibition of the lipase by FAs due to their defective removal from the plasma. We conclude that CD36 is important for both secretion and clearance of intestinal lipoproteins. CD36 deficiency results in hypertriglyceridemia both in the postprandial and fasting states and in humans may constitute a risk factor for diet-induced type 2 diabetes and cardiovascular disease.
\end{abstract}

\section{Introduction}

CD36 is a multiligand scavenger receptor expressed on the plasma membrane by many cells such as platelets, monocytes, and adipocytes, as well as epithelial cells of the retina, mammary gland, and small intestine (reviewed in ref. 1). CD36 appears to be involved in many cellular functions such as cell adhesion (2), angiogenesis (3), and lipoprotein binding/endocytosis (4). The protein is also known as fatty acid (FA) translocase, or FAT, since it is implicated in facilitating uptake of long-chain FAs (5).

Genetic ablation of the Cd36 gene in mice results in impaired FA uptake by numerous tissues (5). Fasting plasma triacylglycerol (TAG) levels are also increased (6) and redistributed from LDL to VLDL. On the other hand, muscle-targeted CD36 overexpression reduces plasma FA and VLDL-associated TAG levels (7). In the spontaneously hypertensive rat, mutations in the $C d 36$ gene were identified and linked to the hypertriglyceridemia, high FA levels, and insulin resistance that are characteristic of this model (8-10), and these symptoms are improved by expression of the WT protein. In summary, the findings from studies on rodents document a role for CD36 in the metabolism of FAs and TAGs.

Nonstandard abbreviations used: BMIPP, $\left[{ }^{125} \Gamma\right] 15$-(p-iodophenyl)-3-(R,S)-methyl pentadecanoic acid; Dgat, diacylglycerol $O$-acyltransferase; ER, endoplasmic reticulum; FA, fatty acid; HL, hepatic lipase; IPPA, [225] 15-p-iodophenyl pentadecanoic acid; KRH, Krebs-Ringer HEPES; LPL, lipoprotein lipase; TAG, triacylglycerol; triton, Triton WR-1339.

Conflict of interest: The authors have declared that no conflict of interest exists.

Citation for this article: J. Clin. Invest. 115:1290-1297 (2005).

doi:10.1172/JCI200521514
In humans, CD36 deficiency was first identified in patients with refractoriness to multiple platelet transfusions (11) and is relatively common (2-7\%) in persons of Asian and African descent $(12,13)$. CD36-deficient subjects may present with high blood pressure and insulin resistance (14) as well as plasma lipid abnormalities. With fasting, high plasma TAG and reduced HDL-associated cholesterol levels were reported by Miyaoka et al. (14), while Yanai et al. observed elevated levels of LDL-associated cholesterol (12). In white people, common polymorphisms in the CD36 gene are associated with elevated blood FA levels (15). Kuwasako et al. further reported increased plasma levels of TAGs and apoB-48 in the postprandial state (16). Thus, CD36 deficiency or mutations in the CD36 gene are associated with abnormal plasma lipid levels in humans.

The role of CD36 in modulating the uptake and utilization of intestine-derived lipids is currently unknown. In humans and mice, CD36 is detected in epithelial cells of the small intestine along the gastrocolic and crypt-to-villus axes in a pattern paralleling that of other proteins implicated in FA uptake, such as intestinal and liver FA-binding proteins (17-19). Immunofluorescence studies revealed a polarized distribution of CD36 along the apical, brush border membranes, the primary site of intestinal FA uptake (19). To directly examine whether CD36 facilitates the utilization of dietary FAs, we measured FA uptake and processing by intestines of WT and CD36-deficient mice. Intestinal lipid secretion into lymph and appearance of TAGs in plasma were determined following intragastric gavage with FAs or olive oil. 

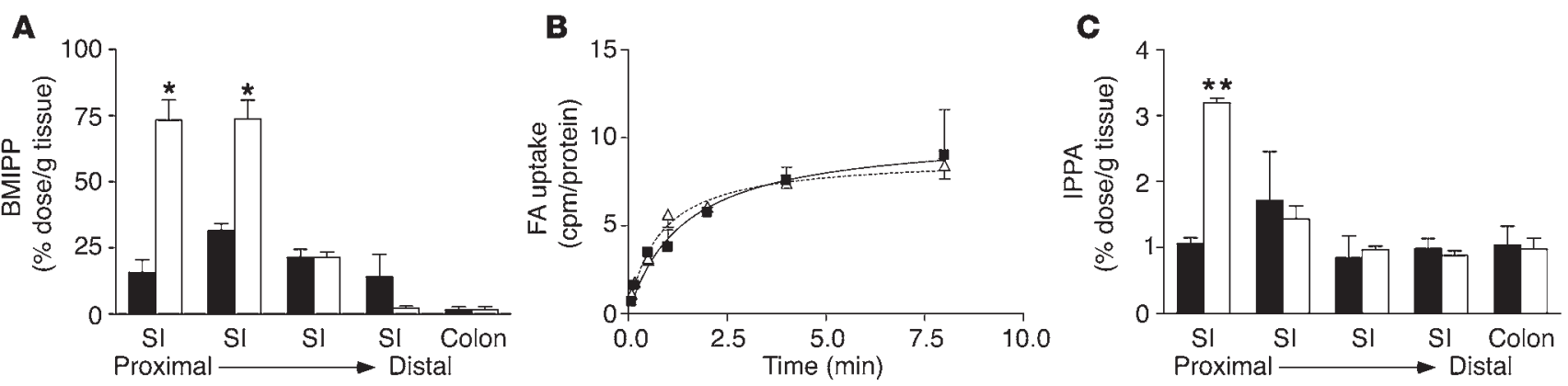

Figure 1

Accumulation of FA analogs in the small intestine after gavage with olive oil. (A) BMIPP levels 2 hours after gavage. WT (black bars) and CD36null (white bars) mice $(n=3)$ were administered BMIPP by gavage with olive oil. After 2 hours, intestinal segments $(\mathrm{SI})$ were harvested from the mice and flushed with PBS. Levels of the FA analogs were determined by $\gamma$-ray spectroscopy. ${ }^{\star} P<0.005$. (B) FA uptake in primary enterocytes. Enterocytes were isolated from WT (filled squares) and CD36-null mice (open triangles). FA uptake at the indicated times was determined as described in Methods. (C) IPPA levels 72 hours after gavage. WT (black bars) and CD36-null (white bars) mice $(n=3)$ were administered IPPA by gavage with olive oil. After 72 hours, intestinal segments were harvested from the mice and flushed with PBS. Levels of the FA analogs were determined by $\gamma$-ray spectroscopy. ${ }^{\star \star} P<0.0001$.

\section{Results}

Lipids accumulate in the proximal small intestine of CD36-deficient mice in vivo. $\left[{ }^{125} \mathrm{I}\right] 15-(p$-iodophenyl)-3-( $R, S)$-methyl pentadecanoic acid (BMIPP) and [ $\left.{ }^{125} \mathrm{I}\right] 15$ - $p$-iodophenyl pentadecanoic acid (IPPA) are FA analogs used to study FA uptake in humans (20) and mice (5). Incorporation of these analogs into cellular lipids is similar to that of native long-chain FAs. BMIPP, which is 3-methylated, is slowly oxidized and retained longer in tissues, thus providing more sensitivity, while the straight-chain IPPA is rapidly oxidized like native FA. We used these analogs to study the effects of CD36 deficiency on intestinal FA uptake and secretion in vivo following intragastric gavage. BMIPP levels 2 hours after gavage were significantly increased in proximal segments of the small intestine of CD36-null mice but unaffected in the distal segments and colon (Figure 1A). These data suggested either increased FA uptake or reduced FA secretion by intestines of CD36-null mice.

As shown in Figure 1B, uptake of FAs was not altered in isolated CD36-null enterocytes. Fecal TAG levels were comparable in WT and CD36-null mice fed a high-fat diet (3.15 \pm 0.51 vs. $2.76 \pm 0.62 \mu \mathrm{g} / \mathrm{mg}$ of feces; $n=7 ; P=0.31$ ). In addition, these data indicated no global defect in fat absorption in CD36deficient mice, consistent with the study by Goudriaan et al. (21) and with the report that other proteins such as FA transfer protein-4 (FATP4) may facilitate FA uptake by enterocytes (22). Thus, we hypothesized that intestinal lipid output may be impaired, leading to accumulation of the FA analog. To test this, we administered IPPA by gavage, and radioactivity in the gastrointestinal segments was measured at an extended time point (72 hours after gavage) to identify alterations in lipid output as opposed to uptake. As shown in Figure 1C, IPPA levels were elevated in the proximal small intestine following the pattern observed with BMIPP.

To determine whether the apparent defect in intestinal lipid output affected intracellular TAG levels under conditions of high-fat loading, we examined intestinal sections obtained from mice fed a high-fat diet for 6 weeks using light microscopy. Sections from CD36-null mice showed abundant oil red O staining, while WT controls did not (Figure 2). In the CD36-deficient sections, staining was concentrated within the enterocytes of the upper villi and absent from the submucosa or serous coat.
CD36 is required for efficient intestinal lipid secretion. To examine lipid secretion directly, we equipped mice with duodenal and intestinal lymph duct fistulae (23). A lipid emulsion of $\left[{ }^{3} \mathrm{H}\right] \mathrm{TAG}$ was then infused via the duodenal fistula, and we determined secretion of TAGs into the mesenteric lymph by monitoring glycerol mass in the lymph fistula. As shown in Figure 3A, lymph TAG recovery in CD36-null mice reached $23 \%$ of the infusate at 6 hours compared with $67 \%$ in WT mice. ${ }^{3} \mathrm{H}$ recovery in the lymph mirrored the TAG mass (at 6 hours: WT, $56.0 \% \pm 14.0 \%$; CD36-null, $23.5 \% \pm 4.5 \%$; $P=0.006)$. Together, these data indicated that lipid secretion from the intestine is significantly impaired in CD36-null mice.

We next examined whether the defect in TAG secretion in CD36deficient intestines reflected impaired FA delivery to the endoplas-
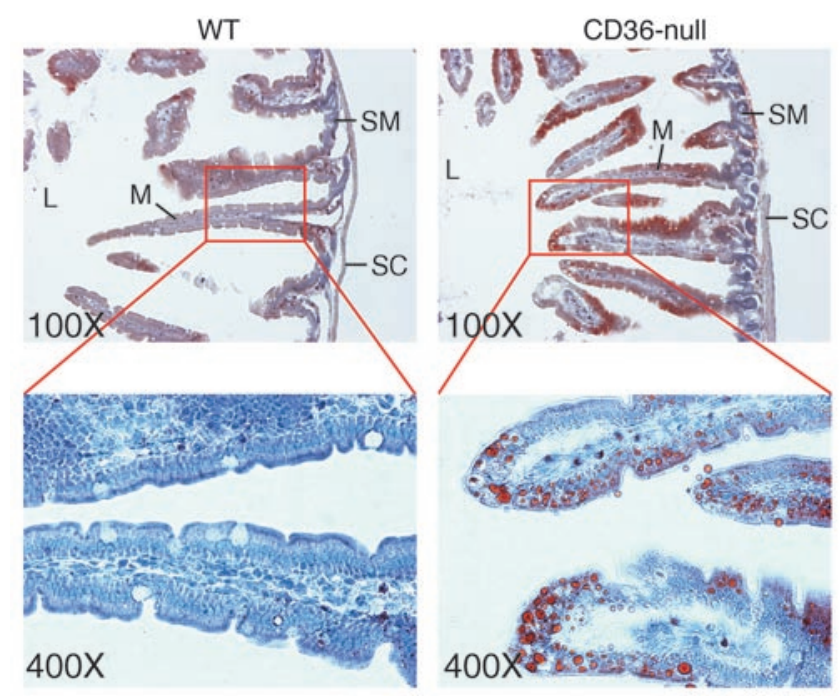

\section{Figure 2}

Accumulation of neutral lipid in intestine from mice fed a high-fat diet. WT and CD36-null mice were fed a high-fat diet ad libitum for 6 weeks. Intestinal segments were fixed in paraformaldehyde/sucrose and prepared for histology using standard techniques. Frozen sections were prepared, stained with oil red $\mathrm{O}$ and hematoxylin, and visualized by light microscopy at the indicated magnifications. L, intestinal lumen; M, mucosa; SC, serous coat; SM, submucosa. 
A

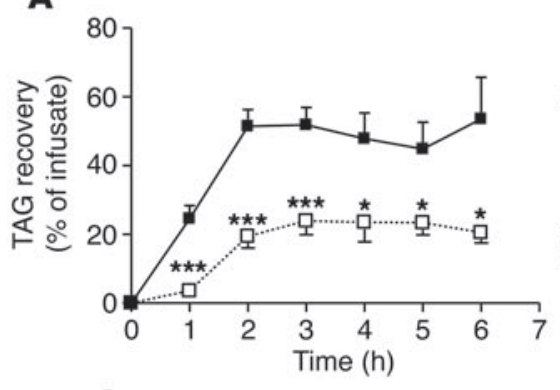

C

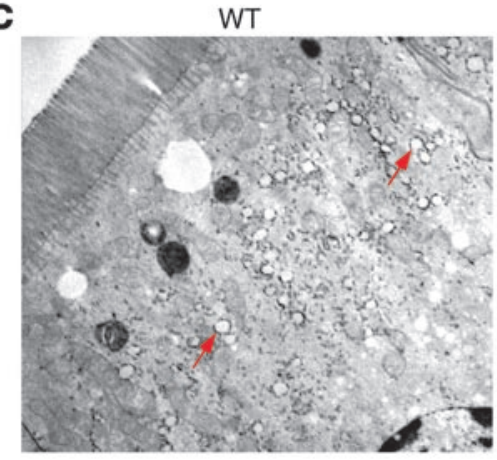

B
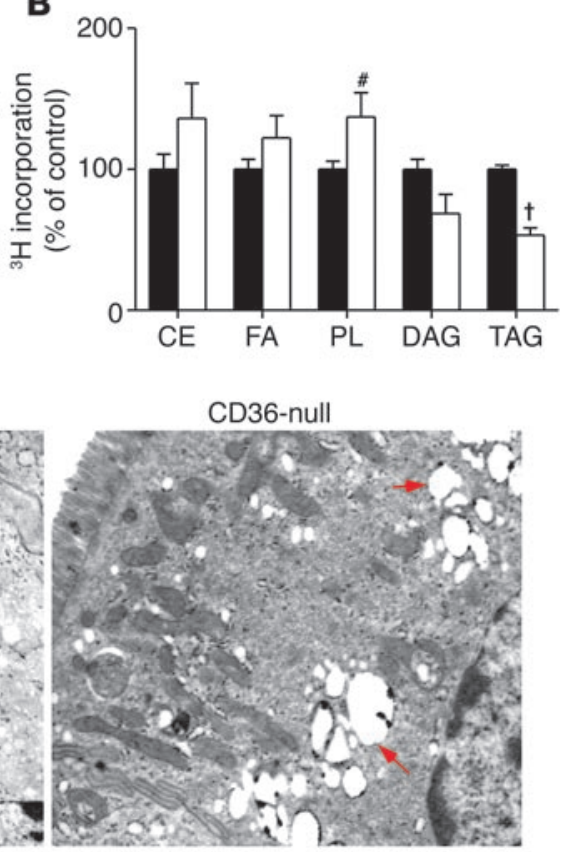

\section{Figure 3}

Lipid trafficking in the intestine. (A) Duodenal and intestinal lymph duct fistulae were surgically implanted in anesthetized WT (filled squares) and CD36-null (open squares) mice $(n=4-6)$. Following duodenal infusion of the lipid emulsion, lymph was collected from the lymph duct fistula. The amount of TAG mass recovered per hour in the lymph (expressed as a percentage of the infusate) was determined by scintillation spectroscopy. ${ }^{\star} P<0.05$; ${ }^{* * *} P<0.001$. (B) Primary enterocytes from anesthetized WT (black bars) and CD36-null (white bars) mice were prepared and pulsed with a lipid mixture containing $\left[{ }^{3} \mathrm{H}\right]$ oleic acid. The relative incorporation of ${ }^{3} \mathrm{H}$ into microsomal lipids was determined. The combined data from 2 experiments ( $n=4$ plates per experiment) is shown. CE, cholesteryl ester; DAG, diacylglycerol; PL, phospholipid. ${ }^{\#} P=0.03 ; \uparrow P<0.00001$. (C) Fixed segments of the proximal intestine were stained with uranyl acetate and embedded using standard methods. Ultrathin sections were prepared, stained with Reynolds' lead citrate, and examined under a Hitachi $\mathrm{H}-600$ electron microscope. Red arrows indicate the positions of lipid droplets.

mic reticulum (ER), which contains the TAG synthetic enzymes and chylomicron assembly machinery. Primary enterocytes obtained from either WT or CD36-null mice were pulsed with micellar $\left[{ }^{3} \mathrm{H}\right]$ oleic acid. Microsomal fractions were then prepared, and incorporation of the label into the major lipid classes was determined. As shown in Figure 3B, recovery of ${ }^{3} \mathrm{H}$ into microsomal TAGs was reduced by $47 \%$ in CD36-null enterocytes, which indicated a defect in microsomal TAG synthesis from exogenous FAs. Incorporation into diacylglycerol (DAG) also trended lower in CD36-null enterocytes $(P=0.07)$, and the DAG/TAG ratio was unaltered and similar to that observed in WT cells (data not shown). The lack of change in this ratio argued against impairment being at the level of DAG conversion to TAG by the enzyme diacylglycerol $O$-acyltransferase (Dgat). In line with this, abundance of mRNA for Dgat 1 and Dgat2, measured by quantitative PCR, was not altered by CD36 deficiency (data not shown). In contrast to incorporation into TAG, ${ }^{3} \mathrm{H}$ into cholesteryl esters trended higher in CD36-null enterocytes, and a significant increase was measured for phospholipids. FA/TAG trafficking was also evaluated by electron microscopy of the proximal intestine after duodenal infusion of triolein. Large and centrally located lipid vacuoles were observed in CD36-deficient enterocytes, while WT cells exhibited mostly small and peripheral droplets (Figure 3C, arrows). These data were consistent with abnormal intracellular targeting of the FAs in CD36-deficient enterocytes.

Clearance of TAG-rich lipoproteins is defective in CD36-deficient mice. Since the fistula studies indicated reduced intestinal lipid secretion in CD36-null mice, the net effect of an olive oil gavage on the postprandial rise in plasma TAG concentration was examined. In WT mice, the level of plasma TAGs (determined by measuring plasma glycerol) was only modestly increased after an olive oil gavage (Figure 4A). In contrast, CD36-null mice displayed significantly higher plasma TAG levels, which persisted for more than 4 hours. These findings were surprising and inconsistent with a reduction of intestinal lipid secretion. To clarify this issue, we tested whether a decrease in chylomicron clearance might be masking the reduced intestinal lipid output from CD36-null mice. Thirty minutes prior to an intragastric olive oil gavage, mice were administered saline or Triton WR-1339 (triton) by i.p. injection. Three hours after the gavage, the level of plasma TAGs was dramatically increased in WT mice given triton (Figure 4B), which indicated that plasma lipase activity and thus chylomicron clearance were blocked. In contrast, plasma TAG levels in CD36-null mice were similar whether or not triton was administered and were comparable to those of WT mice that received triton. Also, changes in hepatic VLDL secretion during the course of the experiment could not explain the differences in plasma TAG levels. Plasma TAG levels in triton-treated fasted mice were comparable in both genotypes (Figure 4C). These data strongly suggested that CD36 deficiency interferes with lipase activity in vivo.

Chylomicrons are secreted from the intestine as large particles, which become smaller through the lipolytic actions of lipoprotein lipase (LPL); thus, chylomicron size can provide a measure of lipase activity in vivo. Chylomicrons were isolated from the plasma of mice 3 hours after an olive oil gavage, and their size was measured by negative-staining electron microscopy. Chylomicrons from WT mice were primarily (97\%) less than $101 \mathrm{~nm}$ in diameter (Figure 4D), while the majority of chylomicrons from CD36-null mice (78\%) were 201-700 $\mathrm{nm}$ in diameter, which further indicated a lipase defect.

We also examined the plasma levels of the major apolipoproteins involved in chylomicron assembly and clearance. $A p o B$ is the main protein component of chylomicrons, and elevated plasma TAG levels are typically associated with elevated plasma apoB levels. As expected, apoB concentrations tended to be higher in the plasma of olive oil-fed CD36-null mice compared with WT mice $(118.2 \pm 34.8$ vs. $68.0 \pm 5.7 \mathrm{mg} / 1 ; n=5 ; P=0.09)$, even though fasting apoB levels were not increased in CD36-null mice (data not shown). ApoE binds TAG-rich lipoproteins and is required for the clearance of chylomicron remnants by the liver. To measure both the levels of apoE and its association with postprandial lipids, we isolated the VLDL/chylomicron fractions from the plasma by size-exclusion chromatography. As shown in Figure 5, relative apoE levels in these 

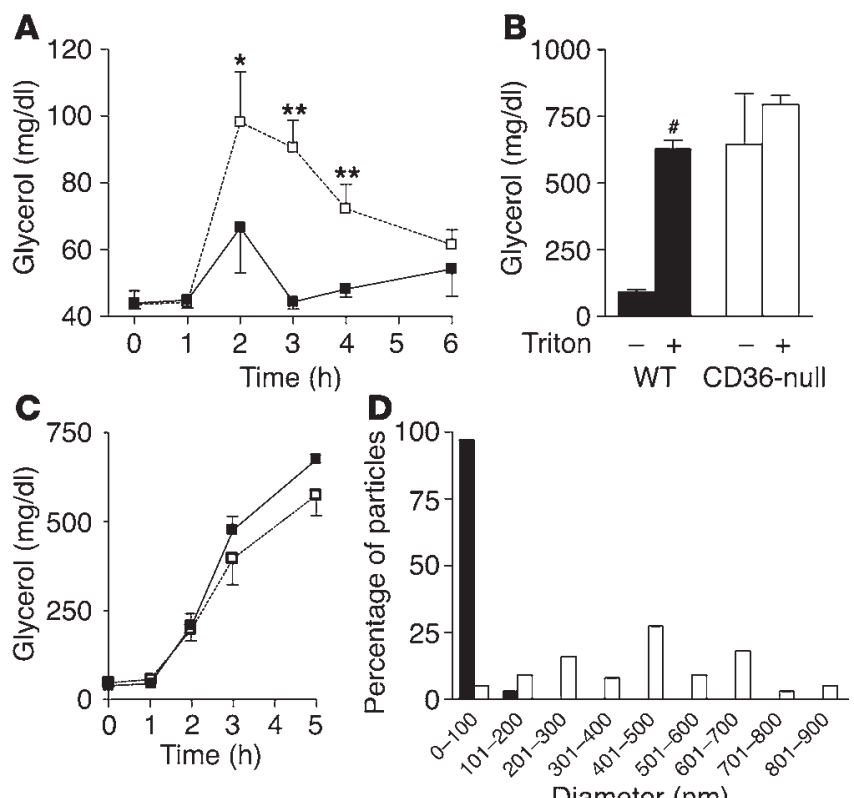

fractions were higher in CD36-null mice independent of the dietary status, and the highest apoE levels covaried with plasma TAG levels. An oral fat load elevated the apoE level in these fractions in both WT and CD36-null mice.

CD36-deficient mice have normal postheparin lipase activity. Postheparin LPL and hepatic lipase (HL) activity in plasma did not differ between WT and CD36-null mice (Figure 6A). Cell-associated lipase activity was also measured in heart tissue collected in the absence of heparin, and no decrease in LPL activity was observed (data not shown). LPL activity can be inhibited by plasma FAs (24). CD36null mice have elevated fasting plasma FA levels $(6,25)$, but it is not known whether FA levels also rise postprandially and interfere with LPL activity and lipoprotein clearance. As shown in Figure 6B, plasma FA levels measured after olive oil gavage were significantly higher in CD36-null mice and were tightly correlated with plasma TAG levels (Figure 6C) $(r=0.7452 ; P=0.0001 ; n=19)$.

Apoc3 mRNA abundance is altered in CD36-null mice. LPL activity can be modulated by apolipoproteins, particularly apoC-I, apoC-II, and apoC-III. The abundance of the mRNAs - Apoc1, Apoc2, and $A p o c 3$, respectively - encoding these proteins was measured in the proximal intestine (Apoc2/Apoc3) and liver (Apoc1/Apoc2/Apoc3). No significant differences in the abundance of the Apoc1 or Apoc2 mRNAs were detected between WT and CD36-null mice following olive oil gavage (Figure 7). On the other hand, the mRNA encoding apoC-III, a potent LPL inhibitor, was significantly increased in CD36-null mice in both intestine and liver. Apoc3 mRNA levels in the intestine correlated with plasma TAG levels $(r=0.4141$; $P=0.039, n=19)$, and a similar trend was detected in the liver $(r=0.3720 ; P=0.059 ; n=19)$.

\section{Discussion}

The role of CD36 in FA uptake in muscle and adipose tissue has been well documented both in vitro and in vivo. The current study illustrates for the first time to our knowledge that CD36 is important for optimal utilization of dietary FAs and triglycerides. In CD36-null mice, secretion of lipid into the mesenteric lymph is significantly reduced, but this effect is masked in vivo, as CD36

\section{Figure 4}

Plasma TAG metabolism in vivo. (A) Postprandial plasma TAGs. WT (filled squares) and CD36-null (open squares) mice $(n=4)$ received an intragastric gavage of olive oil $(16.5 \mu \mathrm{l} / \mathrm{g})$. Plasma was collected at the indicated time points and TAG levels measured. ${ }^{*} P<0.05$; ${ }^{* \star} P<0.001$. (B) Effect of triton on postprandial plasma TAGs. WT (black bars) and CD36-null (white bars) mice $(n=3)$ received i.p. injections of saline or triton 30 minutes before an intragastric gavage of olive oil $(16.5 \mu \mathrm{l} / \mathrm{g})$ was administered. Plasma was collected 3 hours after injection and TAG levels measured. $\# P<0.0001$. (C) Effect of triton on fasting plasma TAGs. WT (filled squares) and CD36-null (open squares) mice $(n=4)$ received i.p. injections of triton. Plasma was collected at the indicated time points and TAG levels measured. (D) Chylomicron size after an oral fat load. Chylomicrons were isolated from the plasma of WT (black bars) and CD36-null (white bars) mice 3 hours after an intragastric gavage of olive oil $(16.5 \mu \mathrm{l} / \mathrm{g})$. Particle size was determined by negative-staining electron microscopy.

also appears to regulate the catabolism of TAG-rich chylomicrons from the plasma.

Enterocyte CD36 may target the FAs to the TAG secretion pool. The reduced TAG output into intestinal lymph in CD36-null mice appeared to reflect mis-targeting of the FAs. Fat feeding or oral administration of FA analogs resulted in accumulation of neutral lipid in CD36-deficient enterocytes. However, these cells also exhibited reduced incorporation of exogenous FAs into microsomal TAGs. Enteric neutral lipid has been proposed to exist in at least 2 pools: a cytosolic storage pool and a microsomal secretion-coupled pool (reviewed in ref. 26). It is thought that de novo synthesis in the ER lumen is the major source of chylomicron TAGs. The data strongly suggest that CD36 deficiency results in impaired FA targeting to the ER for chylomicron

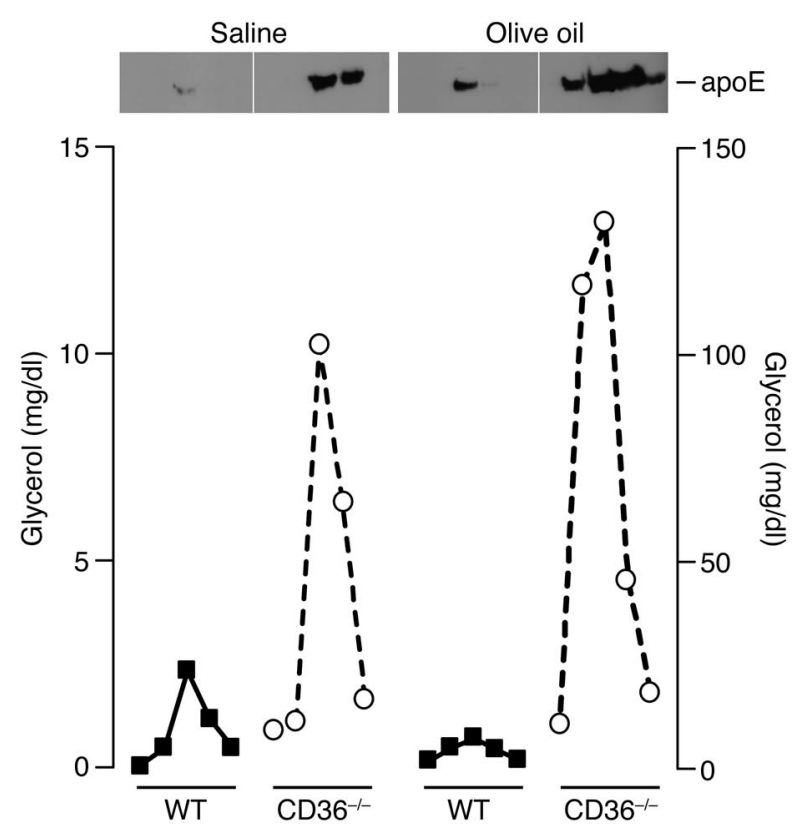

Figure 5

TAG-associated apoE from plasma. WT (filled squares) and CD36-null (open circles) mice $(n=3)$ received an intragastric gavage of saline or olive oil $(16.5 \mu \mathrm{l} / \mathrm{g})$. After 2 hours, plasma was collected and pooled and the VLDL/chylomicron fraction isolated by size-exclusion chromatography. Plasma TAG and apoE levels were measured in each fraction. 
A

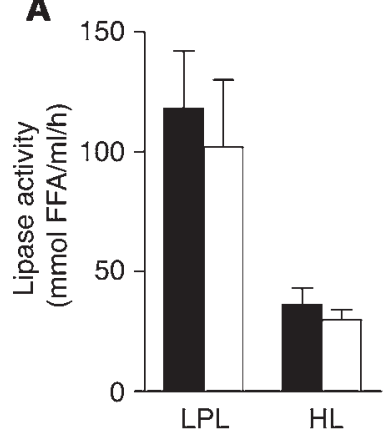

B

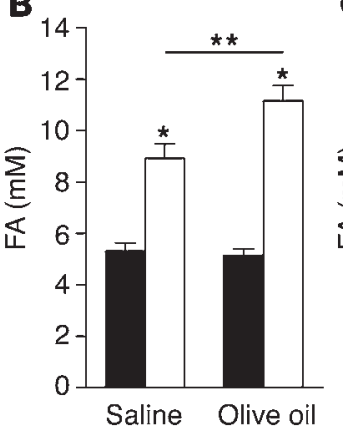

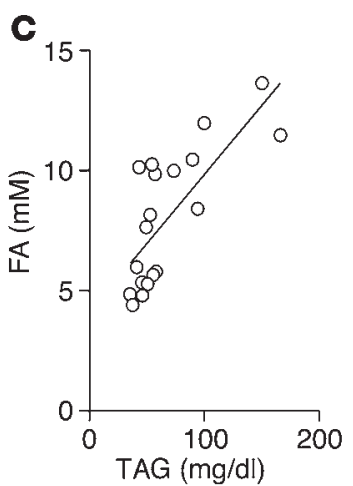

\section{Figure 6}

Postheparin lipase activity and plasma FA levels. (A) LPL and HL activity. WT (black bars) and CD36-null (white bars) mice $(n=5)$ received i.p. injections of heparin. After 30 minutes, plasma was collected and lipase activity measured. (B and C) Plasma FA levels correlate with plasma TAG levels. Fasted WT (black bars) and CD36-null (white bars) mice ( $n=4-6)$ received olive oil $(3.5 \mu \mathrm{l} / \mathrm{g})$ by gavage. Plasma was harvested 2-3 hours after gavage. Plasma FA levels were measured (B) and correlated with plasma TAG levels $(\mathbf{C}) .{ }^{\star} P<0.001 ;{ }^{* \star} P<0.02$. assembly. The mechanism by which CD36 would target dietary long-chain FAs to the secretion-coupled pool in the ER is completely unknown but may involve intracellular trafficking of the protein, which has been shown to cycle between the cell surface and intracellular membranes (27). A working hypothesis is that following an oral fat load, CD36-rich membrane vesicles help shuttle FAs to ER enzymes required for TAG synthesis (Figure 8). For example Dgat1 (28) has been postulated to localize on the luminal side of the ER (29), and FA delivery to Dgat1 and other esterification enzymes could become limiting.

Defect in tissue FA clearance inhibits lipolysis of blood TAGs. Several lines of evidence indicate that CD36 deficiency results in defective lipoprotein lipolysis by LPL. Under fasting conditions, the level of VLDL-associated TAGs is elevated in mice that lack CD36, while LDL-associated TAGs are practically absent (6). Olive oil gavage greatly increased plasma TAG levels in CD36-null mice as compared with WT mice. Triton, a potent inhibitor of LPL and HL, caused a 6-fold increase in the level of plasma TAGs in WT mice following olive oil gavage but had no significant effect in CD36-null mice. In addition, WT and CD36-null mice had similar plasma TAG levels following triton administration in the absence of an olive oil gavage, which indicates that hepatic TAG secretion was not a contributing factor. Also, in the absence of triton, chylomicrons remained large in CD36-null mice, unlike particles in WT mice. All the above data support the interpretation that CD36 deficiency impairs chylomicron catabolism. Analysis of plasma apolipoprotein levels indicated that chylomicrons from WT and CD36-null mice had appropriate levels of apoB and apoE. It is thus unlikely that the decreased catabolism was due to the particles having abnormal structural components.

Plasma LPL and HL and cell-associated lipase activity were not reduced in CD36-deficient mice, which implied that local factors were modulating lipase activity in vivo. FAs can displace LPL from the cell surface, thereby reducing its activity $(24,30,31)$. Conversely, promotion of tissue FA esterification and trapping with acylation-stimulating protein enhances adipocyte LPL activity (32). CD36 is required for efficient tissue FA uptake (5), so inhibition of LPL activity in CD36-deficient mice likely results from the lack of peripheral FA removal. In line with this, a tight correlation was observed between plasma TAG and FA levels in WT and CD36-null mice following an oral fat load.

Additional factors may contribute to or account for the reduced plasma TAG hydrolysis in vivo. Induction of Apoc3 gene expression in the liver and intestine may increase plasma apoC-III levels and thus inhibit LPL activity. In addition, CD36 is a receptor for many lipoprotein classes in vitro (33) and may serve as a docking site for lipoproteins on the cell surface (as suggested in ref. 12), which can then be lipolyzed by cell-associated LPL.

The data presented herein suggest that CD36 regulates intestinal lipid secretion and peripheral clearance of intestinederived lipoproteins (Figure 8), thus linking dietary fat intake and utilization. A high-fat diet increases Cd36 mRNA abundance in the intestine as well as in the heart and adipose tissue $(19,34,35)$. This coordinate regulation favors intestinal TAG synthesis/chylomicron secretion and peripheral chylomicron hydrolysis via increased FA clearance and elevated LPL activity. It may also promote FA oxidation by muscle (36) and TAG storage by adipose tissue (35), processes that appear to be rate limited by FA uptake and CD36 levels.

Postprandial lipemia in buman CD36 deficiency. CD36-deficient humans exhibit abnormalities of plasma lipid profiles including higher postprandial TAG levels $(12,14,16)$. Based on the data presented here, human dyslipidemia during CD36 deficiency may result from defects in the clearance of both FAs and TAGs in both the fed and fasted conditions. Defective clearance of dietary TAGs has not been documented in CD36-deficient humans but would be implied by the observed increase of plasma apoB-48 levels (16).

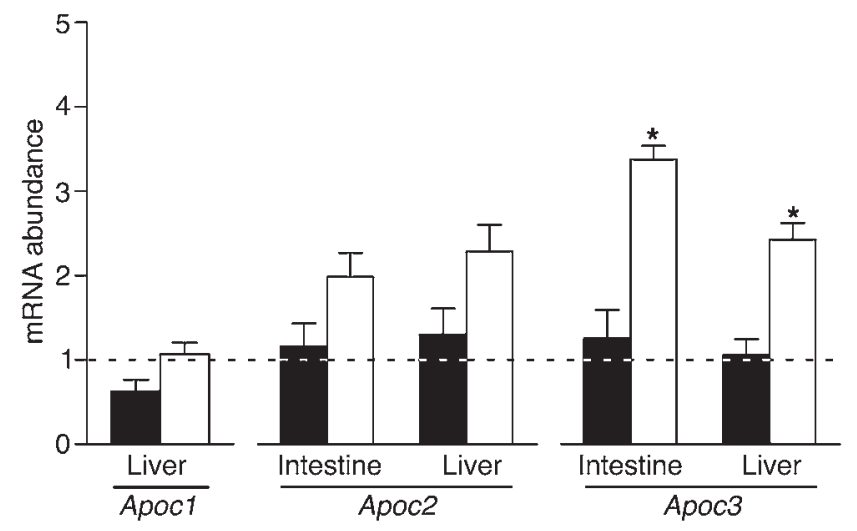

\section{Figure 7}

Intestinal and hepatic Apoc mRNA levels. Fasted WT (black bars) and CD36-null (white bars) mice $(n=4-6)$ received olive oil $(3.5 \mu \mathrm{l} / \mathrm{g})$ by gavage. Intestinal and hepatic tissues were harvested 2-3 hours after gavage and used to prepare total RNA. Hepatic and intestinal Apoc1, Apoc2, and Apoc3 mRNA abundance was measured by quantitative RT-PCR and related to that of cyclophilin. For each gene, the abundance in saline-treated WT mice was taken as 1 (dashed line). ${ }^{*} P<0.001$. 


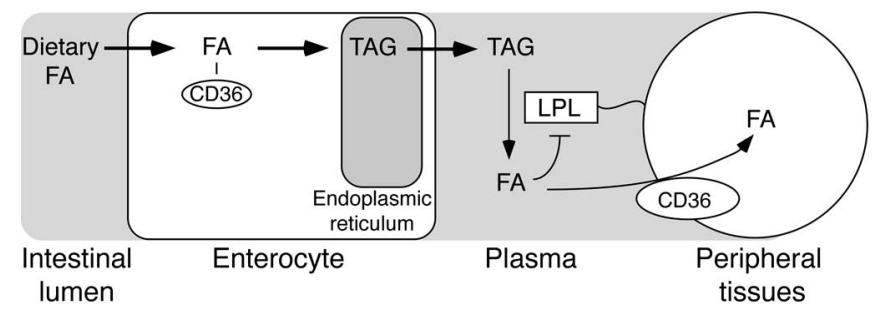

Figure 8

CD36 and dietary FA utilization. In the presence of CD36, FAs are targeted to a secretion-coupled pool within the ER and efficiently packaged into TAG chylomicrons. In the plasma, LPL acts to liberate the FAs from chylomicron-associated TAGs. Clearance of released FAs by peripheral tissues via CD36 prevents feedback inhibition of $\operatorname{LPL}(33,34)$. Coordinated upregulation of CD36 expression by dietary FAs (37) would enhance both FA absorption and peripheral utilization.

Since apoB-48 in humans is only produced in the intestine, a high level of plasma apoB-48 is consistent with impaired chylomicron clearance and TAG hydrolysis. Hypertriglyceridemia has been linked to the etiology of type 2 diabetes and atherosclerosis (37), and it is important to examine whether CD36 deficiency alters susceptibility to these pathologies, particularly when they result from high-fat diets. In this context, Ma et al. (15) reported that a common haplotype at the CD36 locus was associated with an increased risk of cardiovascular disease in type 2 diabetes.

Diabetic subjects exhibit extended postprandial lipemia, and as our data suggest, a potential mechanism for this abnormality is the high level of plasma FA that is common in diabetes (38). Our findings further emphasize the importance of treatments aimed at improving tissue FA clearance in patients with diabetes. Thus, enhancing tissue FA uptake by upregulation of CD36 may mediate a significant component of the beneficial effects of thiazolidinediones on blood lipids and energy utilization (38-40).

\section{Methods}

Animals. CD36-null mice backcrossed to the C57BL6/J background (5) and WT (C57BL6/J) mice (8-12 weeks of age) were maintained on standard rodent chow (unless otherwise indicated) under a normal light-dark cycle. All experimental procedures were approved by the Institutional Animal Care and Use Committee of the Division of Laboratory Animal Resources at the State University of New York at Stony Brook. Primary enterocytes were prepared from intestinal segments of starved mice as described in ref. 41.

Intestinal lipid analysis. For in vivo studies, mixtures of FA analogs were prepared as follows: $40 \mu \mathrm{Ci}$ of either BMIPP or IPPA (Oak Ridge National Laboratory) was evaporated under $\mathrm{N}_{2(\mathrm{~g})}$ in a glass vial and resuspended in $2 \mathrm{ml}$ warm olive oil by gentle sonication. Triton $(500 \mathrm{mg} / \mathrm{kg})$ was administered to fasted (8-12 hours) mice by i.p. injection, and 30 minutes later, the FA analog/olive oil mixture $(6 \mu \mathrm{l} / \mathrm{g})$ was administered by intragastric gavage. Mice were sacrificed 2 or 72 hours after gavage as indicated in Figure 1, and 4 intestinal segments of equal length plus the colon were collected, salinewashed, and associated radioactivity measured using $\gamma$-ray spectroscopy.

For in vitro studies in primary enterocytes, $\left[{ }^{14} \mathrm{C}\right]$ palmitate:BSA complexes were prepared in a solution of $160 \mu \mathrm{M}$ sodium palmitate (SigmaAldrich), $5 \mathrm{mM}$ glucose, and $40 \mu \mathrm{M}$ FA-free albumin (Fraction V; SigmaAldrich) in Krebs-Ringer HEPES (KRH) buffer ( $\mathrm{pH} 7.4$ ). While stirring, we added $0.5 \mu \mathrm{Ci} / \mathrm{ml}$ of $\left[1-{ }^{14} \mathrm{C}\right]$ palmitic acid $(55 \mathrm{mCi} / \mathrm{mmol}$; ICN Biomedicals Inc.) and unlabeled palmitic acid to produce a 1:1 solution.
Primary enterocytes $(25 \mu \mathrm{l})$ resuspended in $\mathrm{KRH}$ were added to a $25-\mu \mathrm{l}$ aliquot of $\left[{ }^{14} \mathrm{C}\right]$ palmitate:BSA complex for the indicated times. The uptake reaction was stopped by the addition of ice-cold stop solution $(200 \mu \mathrm{l}$; $300 \mu \mathrm{M}$ phloretin [Sigma-Aldrich] in KRH) and immediately overlaid on an ice-cold plug of Ficoll-Paque (Amersham Biosciences; diluted 6-fold in PBS) in $6 \times 44 \mathrm{~mm}$ microcentrifuge tubes. Cells were separated from free $\left[{ }^{14} \mathrm{C}\right]$ palmitate by centrifugation at $12,000 \mathrm{~g}$ for 1 minute. The cell pellet was cut from the bottom of the tube, incubated overnight in scintillation fluid, and quantitated by scintillation spectroscopy. Background uptake and/or nonspecific cell association of the $\left[{ }^{14} \mathrm{C}\right]$ palmitate were routinely subtracted from results and were determined by adding the enterocytes to ice-cold mixtures of $\left[{ }^{14} \mathrm{C}\right]$ palmitate:BSA and stop solution. The samples were then processed as described above. No difference in background uptake was observed between enterocytes isolated from WT and CD36-null mice (data not shown).

To measure FA partitioning, primary enterocytes were plated and pulsed with a labeling mixture (DMEM containing $0.4 \mathrm{mM}$ sodium taurocholate, $0.52 \mathrm{mM}$ sodium taurodeoxycholate, $0.29 \mathrm{mM}$ phosphatidylcholine, $0.45 \mathrm{mM}$ oleic acid, $0.16 \mathrm{mM}$ 1-oleoyl-rac-glycerol, $0.05 \mathrm{mM}$ cholesterol) containing $3.3 \mu \mathrm{Ci} / \mathrm{ml}[9,10-\mathrm{H}(\mathrm{N})]$ oleic acid for 1 hour at $37^{\circ} \mathrm{C} / 5 \% \mathrm{CO}_{2}$. Cells were washed by centrifugation in cold PBS and microsomes were prepared as described in ref. 42. Lipids were extracted using the method of Folch et al. (43), resuspended in chloroform, and separated by TLC in hexane/isopropyl ether/glacial acetic acid 60:40:4 (vol/vol/vol). After the different lipid species were identified according to standards, bands corresponding to the lipids indicated in Figure 1 were scraped, incubated overnight in Scintilene scintillation fluid (Fisher Scientific International Inc.), and quantified by scintillation spectroscopy. The distribution of ${ }^{3} \mathrm{H}$ in the different lipid classes is expressed as a percentage of total cellular counts, with levels in WT cells taken as $100 \%$.

Levels of fecal TAG were determined from mice fed a high-fat, highcarbohydrate diet (F3282; Bioserve) ad libitum. TAGs were extracted as described above and quantitated using the Triglycerides Reagent (Thermo Electron Corp.) and standard curves generated with Glycerol Standard (Sigma-Aldrich).

Lipid secretion into mesenteric lymph. Live animals equipped with duodenal and intestinal lymph duct fistulae (23) were infused with a lipid emulsion containing $13.3 \mathrm{mM}\left[{ }^{3} \mathrm{H}\right]$ triolein, $2.6 \mathrm{mM}$ cholesterol, $2.9 \mathrm{mM}$ egg phosphatidylcholine, and $19.0 \mathrm{mM}$ sodium taurocholate in PBS ( $\mathrm{pH}$ 6.4) at a rate of $0.3 \mathrm{ml} / \mathrm{h}$. Lymph was collected hourly for 6 hours and the recovery of ${ }^{3} \mathrm{H}$ or TAGs (expressed as a percentage of the infusate) determined by scintillation spectroscopy or using Triglycerides Reagent as described above, respectively.

Plasma lipid and lipoprotein analysis. Whole blood was collected from the tail vein into EDTA-treated tubes, and the plasma was isolated by lowspeed centrifugation. TAG levels were measured using the Triglycerides Reagent as described above.

We quantified apoB in plasma using an automated immunoturbidimetric assay (44). For quantification of apoE, the plasma was first fractionated using size exclusion chromatography as described in ref. 45 . We subjected the VLDL/chylomicron fractions to SDS-PAGE on 10\% gels, and after transferring them to nitrocellulose, used $\alpha$-apoE and the ECL kit for immunodetection (Amersham Biosciences).

Lipase activity. Lipase activity in postheparin plasma and in homogenized hearts was analyzed as previously described $(46,47)$.

Histology. Intestines were obtained from mice fed a high-fat, high-carbohydrate diet (F3282; Bioserve) ad libitum for 6 weeks. They were divided into 3 segments of equal length, and the proximal segment was gently flushed with PBS and fixed by 2 overnight incubations at $4{ }^{\circ} \mathrm{C}$, first in $4 \%$ paraformaldehyde and then in $30 \%$ sucrose. Intestinal rings $(0.5 \mathrm{~cm})$ were 
cut from the fixed segments and frozen in cryoembedding media (OCT) for subsequent cryostat sectioning at 7-10 $\mu \mathrm{m}$. Sections were applied to gelatin-coated glass slides, stained with oil red $\mathrm{O}$ and hematoxylin, and mounted with glycerol/water (50:50) media.

Electron microscopy. To measure lipoprotein size, we layered pooled aliquots of sera $(200 \mu \mathrm{l})$ under $600 \mu \mathrm{l} 0.15 \mathrm{M} \mathrm{NaCl}$ and subjected them to centrifugation at $117,320 \mathrm{~g}$ for 30 minutes in a tabletop ultracentrifuge. We removed chylomicrons, subjected them to negative-staining electron microscopy using aqueous uranyl acetate, and viewed them under a Zeiss 902 electron microscope.

To asses FA trafficking, we administered an emulsion of triolein to WT and Cd36-null mice via a duodenal fistula as described above. Proximal intestinal sections were then prepared and visualized essentially as described in ref. 48. Briefly, intestinal tissue was fixed for 2 hours in $2 \%$ glutaraldehyde and for an additional 2 hours in $0.1 \mathrm{M}$ cacodylate buffer. The tissue was then postfixed in $1 \%$ osmium tetroxide and stained en bloc in uranyl acetate. Following dehydration, samples were infiltrated in acetone/ Spurr's mixture and embedded in Spurr's resin. Ultrathin sections were cut on a Reichert Ultracut ultramicrotome, stained with Reynolds' lead citrate, and examined under a Hitachi H-600 electron microscope.

Apolipoprotein $m R N A$ abundance. Fasted (8-12 hours) mice were administered an intragastric gavage of saline or olive oil, and tissues were harvested 2.5 hours later. Total RNA isolation, reverse transcription, and real-time quantitative PCR were performed as previously described (45). Gene-specific, intron-spanning oligonucleotides and SYBR Green I (Sigma-Aldrich) were used to detect amplicon production in a GeneAmp PCR System 9700 (Applied Biosystems). Cyclophilin was used as internal control. For each gene, mRNA abundance in saline-treated WT mice was taken as 1 . The primer sequences were: cyclophilin, 5'-TCCAAAGACAGCAGAAAACTTTCG, 5'-TCTTCTTGCTGGTCTTGCCATTCC; Apoc1, 5'-TGTCCTGATTGTGGTCGTAGCC, 5'-CAAATAGCCTCTAGAGAACCAGGC; Apoc2, 5' - TCCTGGTCATCCTGATGTTGGG, 5' - TGCCTAGCGTAAGTGCTCATGGC; Apoc3, 5'-CTACTCCAGGTACGTAGGTGCC, 5'-TGGTCCTCAGGGTTAGAATCCC.

Statistical analysis. The data shown are mean \pm SEM. Differences between treatment groups were evaluated using Student's $t$ test or ANOVA (Bartlett's test for equal variances and the Bonferroni multiple comparison test. Correlation coefficients $(r)$ were determined using the Pearson product-moment method. $P<0.05$ was considered statistically significant.

\section{Acknowledgments}

We thank Ira Goldberg (Columbia University) for performing the lipase assays and for helpful comments and F.F. Knapp (Oak Ridge National Laboratories) for kindly providing BMIPP and IPPA. Daniel Levine (The Rogosin Institute, New York Presbyterian Hospital-Weill Medical College of Cornell University) kindly measured the plasma apoB levels. This work was supported by NIH grants DK 600220 and DK33301 (to N.A. Abumrad) and grants DK 56260 and HL-38180 (to N.O. Davidson).

Received for publication March 8, 2004, and accepted in revised form February 8, 2005.

Address correspondence to: Nada A. Abumrad, Department of Medicine, Division of Nutrition, Campus Box 8031, Washington University, St. Louis, Missouri 63110, USA. Phone: (314) 747-0348; Fax: (314) 444-3432; E-mail: nabumrad@wustl.edu.
1. Coburn, C.T., and Abumrad, N.A. 2003. Structure-function of CD36 and evidence for its role in facilitating fatty acid transport. In Cellular proteins and their fatty acids in health and disease. A.K. Duttaroy and F. Spener, editors. Wiley-VCH Verlag GmbH. Weinheim, Germany. 3-29.

2. Tandon, N.N., Kralisz, U., and Jamieson, G.A. 1989. Identification of glycoprotein IV (CD36) as a primary receptor for platelet-collagen adhesion. J. Biol. Chem. 264:7576-7583.

3. Dawson, D.W., et al. 1997. CD36 mediates the In vitro inhibitory effects of thrombospondin-1 on endothelial cells. J. Cell Biol. 138:707-717.

4. Endemann, G., et al. 1993. CD36 is a receptor for oxidized low density lipoprotein. J. Biol. Chem. 268:11811-11816.

5. Coburn, C.T., et al. 2000. Defective uptake and utilization of long chain fatty acids in muscle and adipose tissues of CD36 knockout mice. J. Biol. Chem. 275:32523-32529.

6. Febbraio, M., et al. 1999. A null mutation in murine CD36 reveals an important role in fatty acid and lipoprotein metabolism. J. Biol. Chem. 274:19055-19062.

7. Ibrahimi, A., et al. 1999. Muscle-specific overexpression of FAT/CD36 enhances fatty acid oxidation by contracting muscle, reduces plasma triglycerides and fatty acids, and increases plasma glucose and insulin. J. Biol. Chem. 274:26761-26766.

8. Aitman, T.J., et al. 1999. Identification of Cd36 (Fat) as an insulin-resistance gene causing defective fatty acid and glucose metabolism in hypertensive rats. Nat. Genet. 21:76-83.

9. Iritani, N., Fukuda, E., Nara, Y., and Yamori, Y. 1977. Lipid metabolism in spontaneously hypertensive rats (SHR). Atherosclerosis. 28:217-222.

10. Reaven, G.M., Chang, H., Hoffman, B.B., and Azhar, S. 1989. Resistance to insulin-stimulated glucose uptake in adipocytes isolated from spontaneously hypertensive rats. Diabetes. 38:1155-1160.

11. Yamamoto, N., et al. 1990. A platelet membrane glycoprotein (GP) deficiency in healthy blood donors: Naka- platelets lack detectable GPIV (CD36). Blood. 76:1698-1703.

12. Yanai, H., et al. 2000. Human CD36 deficiency is associated with elevation in low-density lipoprotein-cholesterol. Am.J. Med. Genet. 93:299-304.

13. Curtis, B.R., and Aster, R.H. 1996. Incidence of the Nak(a)-negative platelet phenotype in African Americans is similar to that of Asians. Transfusion. 36:331-334.

14. Miyaoka, K., et al. 2001. CD36 deficiency associated with insulin resistance. Lancet. 357:686-687.

15. Ma, X., et al. 2004. A common haplotype at the CD36 locus is associated with high free fatty acid levels and increased cardiovascular risk in Caucasians. Hum. Mol. Genet. 13:2197-2205.

16. Kuwasako, T., et al. 2003. Lipoprotein abnormalities in human genetic CD36 deficiency associated with insulin resistance and abnormal fatty acid metabolism. Diabetes Care. 26:1647-1648.

17. Chen, M., Yang, Y., Braunstein, E., Georgeson, K.E., and Harmon, C.M. 2001. Gut expression and regulation of FAT/CD36: possible role in fatty acid transport in rat enterocytes. Am. J. Physiol. Endocrinol.Metab. 281:E916-E923.

18. Lobo, M.V., et al. 2001. Localization of the lipid receptors CD36 and CLA-1/SR-BI in the human gastrointestinal tract: towards the identification of receptors mediating the intestinal absorption of dietary lipids. J. Histochem. Cytochem. 49:1253-1260.

19. Poirier, H., Degrace, P., Niot, I., Bernard, A., and Besnard, P. 1996. Localization and regulation of the putative membrane fatty-acid transporter (FAT) in the small intestine. Comparison with fatty acid-binding proteins (FABP). Eur. J. Biochem. 238:368-373

20. Nozaki, S., et al. 1999. CD36 mediates long-chain fatty acid transport in human myocardium: complete myocardial accumulation defect of radiolabeled long-chain fatty acid analog in subjects with CD36 deficiency. Mol. Cell. Biochem. 192:129-135.
21. Goudriaan, J.R., et al. 2002. Intestinal lipid absorption is not affected in CD36 deficient mice. Mol. Cell. Biochem. 239:199-202.

22. Gimeno, R.E., et al. 2003. Targeted deletion of fatty acid transport protein-4 results in early embryonic lethality. J. Biol. Chem. 278:49512-49516.

23. Tso, P., Karlstad, M.D., Bistrian, B.R., and DeMichele, S.J. 1995. Intestinal digestion, absorption, and transport of structured triglycerides and cholesterol in rats. Am.J. Physiol. 268:G568-G577.

24. Saxena, U., Witte, L.D., and Goldberg, I.J. 1989. Release of endothelial cell lipoprotein lipase by plasma lipoproteins and free fatty acids. J. Biol. Chem. 264:4349-4355.

25. Hajri, T., Han, X.X., Bonen, A., and Abumrad, N.A. 2002. Defective fatty acid uptake modulates insulin responsiveness and metabolic responses to diet in CD36-null mice. J. Clin. Invest. 109:1381-1389. doi:10.1172/JCI200214596.

26. van Greevenbroek, M.M., and de Bruin, T.W. 1998. Chylomicron synthesis by intestinal cells in vitro and in vivo. Atherosclerosis. 141(Suppl. 1):S9-S16.

27. Coort, S.L., et al. 2004. Enhanced sarcolemmal FAT/CD36 content and triacylglycerol storage in cardiac myocytes from obese zucker rats. Diabetes. 53:1655-1663.

28. Buhman, K.K., et al. 2002. DGAT1 is not essential for intestinal triacylglycerol absorption or chylomicron synthesis. J. Biol. Chem. 277:25474-25479.

29. Washington, L., Cook, G.A., and Mansbach, C.M., II. 2003. Inhibition of carnitine palmitoyltransferase in the rat small intestine reduces export of triacylglycerol into the lymph. J. Lipid Res. 44:1395-1403.

30. Peterson, J., et al. 1990. Fatty acid control of lipoprotein lipase: a link between energy metabolism and lipid transport. Proc. Natl. Acad. Sci. U. S. A. 87:909-913.

31. Bengtsson, G., and Olivecrona, T. 1980. Lipoprotein lipase. Mechanism of product inhibition. Eur. J. Biochem. 106:557-562. 
32. Faraj, M., Sniderman, A., and Cianflone, K. 2004. ASP enhances in situ lipoprotein lipase activity by increasing fatty acid trapping in adipocytes. J. Lipid Res. 45:657-666.

33. Calvo, D., Gomez-Coronado, D., Suarez, Y., Lasuncion, M.A., and Vega, M.A. 1998. Human CD36 is a high affinity receptor for the native lipoproteins HDL, LDL, and VLDL. J. Lipid Res. 39:777-788.

34. Greenwalt, D.E., Scheck, S.H., and RhinehartJones, T. 1995. Heart CD36 expression is increased in murine models of diabetes and in mice fed a high fat diet. J. Clin. Invest. 96:1382-1388.

35. Fabris, R., et al. 2001. Preferential channeling of energy fuels toward fat rather than muscle during high free fatty acid availability in rats. Diabetes. 50:601-608.

36. Belke, D.D., Larsen, T.S., Lopaschuk, G.D., and Severson, D.L. 1999. Glucose and fatty acid metabolism in the isolated working mouse heart. Am.J. Physiol. 277:R1210-R1217.

37. Owens, D. 2003. The extended postprandial phase in diabetes. Biochem. Soc. Trans. 31:1085-1089.
38. Miles, J.M., et al. 2003. Nocturnal and postprandial free fatty acid kinetics in normal and type 2 diabetic subjects: effects of insulin sensitization therapy. Diabetes. 52:675-681.

39. Qi, N., et al. 2002. Pharmacogenetic evidence that cd36 is a key determinant of the metabolic effects of pioglitazone. J. Biol. Chem. 277:48501-48507.

40. Wilmsen, H.M., et al. 2003. Thiazolidinediones upregulate impaired fatty acid uptake in skeletal muscle of type 2 diabetic subjects. Am. J. Physiol. Endocrinol. Metab. 285:E354-E362.

41. Xie, Y., Nassir, F., Luo, J., Buhman, K., and Davidson, N.O. 2003. Intestinal lipoprotein assembly in apobec-1-/- mice reveals subtle alterations in triglyceride secretion coupled with a shift to larger lipoproteins. Am.J. Physiol. Gastrointest. Liver Physiol. 285:G735-G746.

42. Gilham, D., et al. 2003. Inhibitors of hepatic microsomal triacylglycerol hydrolase decrease very low density lipoprotein secretion. FASEB J. 17:1685-1687.

43. Folch, J., Lees, M., and Sloane Stanley, G.H. 1957.
A simple method for the isolation and purification of total lipides from animal tissues. J. Biol. Chem. 226:497-509.

44. Levine, D.M., and Williams, K.J. 1997. Automated measurement of mouse apolipoprotein B: convenient screening tool for mouse models of atherosclerosis. Clin. Chem. 43:669-674.

45. Drover, V.A., and Agellon, L.B. 2004. $\alpha$-hydroxylase gene (CYP7A1) by thyroid hormone in transgenic mice. Endocrinology. 145:574-581.

46. Hocquette, J.F., Graulet, B., and Olivecrona, T. 1998. Lipoprotein lipase activity and mRNA levels in bovine tissues. Comp. Biochem. Physiol. Biochem. Mol. Biol. 121:201-212.

47. Lutz, E.P., et al. 2001. Heparin-binding defective lipoprotein lipase is unstable and causes abnormalities in lipid delivery to tissues. J. Clin. Invest. 107:1183-1192.

48. Sabesin, S.M., and Frase, S. 1977. Electron microscopic studies of the assembly, intracellular transport, and secretion of chylomicrons by rat intestine. J. Lipid Res. 18:496-511. 